\title{
Auditoria de prontuários médicos em unidade de internação hospitalar
}

\author{
Marcilene Cristina Silva Gontijo \\ Administradora graduada pela UNIPAC - Universidade Presidente Antônio Carlos de Carmo do \\ Paranaíba/MG. Pós-graduanda em Auditoria em Sistemas de Saúde pela ASSEVIM/Instituto Passo 1. \\ Patos de Minas/MG
}

marci.cristinasg@yahoo.com.br

\section{Marlúcio Anselmo Alves}

Enfermeiro, mestre em Promoção da Saúde pela UNIFRAN, docente do curso de Pós-graduação latu sensu em Auditoria em Sistemas de Saúde.

marlucioalves@yahoo.com.br

RESUMO: O presente trabalho objetiva identificar o processo de auditoria e como a mesma poderia melhorar a qualidade da assistência especializada prestada aos pacientes sob os cuidados de uma equipe multiprofissional, que prestam serviços na área da saúde. Realizada por meio de uma pesquisa quantitativa e exploratória, através de uma auditoria interna realizada no período de janeiro de 2007 a agosto de 2010 nos 6007 prontuários médicos hospitalares do HSCM, sendo também relacionadas citações bibliográficas que fazem referência ao tema proposto. Os resultados mostram que houve falhas na prescrição e evolução médica quanto ao relato da alta hospitalar, falta do relato de alta do paciente por parte de equipe de enfermagem, coleta incompleta de dados do paciente, ausência de prescrições de SADT como exames laboratoriais e Rx. Porém as falhas na checagem de medicamentos por parte da equipe de enfermagem apresentaram como um dos principais problemas neste estudo, dos quais se acredita ser o ponto mais importante e relevante a ser dissertado. Alguns artigos sugerem que a auditoria seja um instrumento essencial e preciso para o devido controle de perdas, melhoria na assistência prestada ao paciente e um importante campo de atuação para especialistas nas mais diversas áreas profissionais.

PALAVRAS-CHAVE: Auditoria, Análise, Equipe Multiprofissional.

\section{Audit of medical reports in hospital admission unit}

ABSTRACT: This paper aims to identify the audit process and how that could improve the quality of specialized assistance provide for patients under a multiprofessional team who assist in health area. This study was a quantitative and exploratory research, through a inside audit carried out between January 2007 and august 2010 in 6007 hospital medical records of HSCM, besides, bibliographic quotations that refer to the main issue. The results showed there were mistakes on prescription and medical evolution in respect of hospital discharge, lack of description of patients release by nurse team, incomplete data collection, lack of prescription of SADT as exams and $x$-ray. Nevertheless, the medicine checking mistakes by nurse team have emerged as one of the main problems in this study, which is believed to be the most important issue to be discussed. Some papers discuss that the auditory is a important tool to control losses, improve the patient care and a important field for specialists in different professional areas.

KEYWORDS: Audit, Analisys, Multiprofessional team 


\section{INTRODUÇÃO}

O desenvolvimento e o aperfeiçoamento dos profissionais na área de Auditoria em Sistemas de Saúde se tornam primordiais mediante a necessidade de assessoramento da prática devidamente correta de uma equipe de enfermagem, bem como as excessivas perdas e glosas para uma instituição. A auditoria é uma ferramenta precisa, que pode ser explorada e aproveitada pelas instituições no intuito de, cada vez mais, melhorar sua produtividade e evitar perdas, que na maioria das vezes são evitáveis e desnecessárias. Dun e Morgan, apud Kurcgant (1976, p. 106) definem a auditoria como um instrumento da administração utilizado na avaliação da qualidade e do cuidado, sendo a comparação entre a assistência prestada e os padrões de assistência considerados como aceitáveis.

Kron citado apud Ribeiro (1972, p. 91-103) caracteriza a auditoria como avaliação, não somente da eficácia da assistência que o paciente recebe, mas também a integridade e exatidão da demonstração dessa assistência no prontuário.

Importante frisar que a auditoria é uma atividade de coleta de informações para verificar o atendimento aos requisitos especificados, procurando evidências de conformidades, avaliando as necessidades de ações corretivas ou de aperfeiçoamento, não devendo ser confundidas com atividades de supervisão ou inspeção (NBR 9000:2000 -BRASIL, 2000).

Ainda, conforme norma acima citada, a auditoria não tem como objetivo identificar os culpados pela não conformidade e sim, propor soluções para que sejam eliminadas.

"um sistema de revisão e controle com o objetivo de informar o alto escalão da
instituição sobre a eficiência e eficácia dos procedimentos adotados pela instituição,
indicando suas falhas e problemas, servindo para apontar sugestões e soluções,
tendo então um caráter educacional". (CHIAVENATO 1981 apud SILVA, 1990, p.199).

Justifica-se este estudo pela complexidade do tema, onde muito se discute acerca de controle, economia e no cuidado com o paciente. Vários métodos existem, mas poucas pessoas sabem o seu verdadeiro significado ou não procuram segui-los mediante reavaliação da sua ética profissional. Assim acabam não colocando em prática seu trabalho como o deveria, sendo encontradas falhas notáveis que necessitam de verificação, correção, análise e controle.

A metodologia utilizada foi a pesquisa exploratória, de natureza quantitativa pela apresentação e análise dos dados e qualitativa visto que auxiliará no conhecimento e na prática, bem como na compreensão do tema. Os dados foram coletados durante um período de 04 (quatro) meses e 27 (vinte e sete) dias, sendo realizada uma análise em todos os prontuários médicos dos pacientes internados no HSCM, nas clínicas médicas, cirúrgicas, obstétricas e pediátricas dos últimos 04 (quatro) anos. Os dados foram dispostos em gráficos e tabelas para melhor compreensão e discussão.

A elaboração deste trabalho foi baseada em uma Auditoria Interna, visando uma revisão em 6007 prontuários de janeiro de 2007 a agosto de 2010, dos quais foram obtidos o somatório necessário para avaliar o serviço de enfermagem e faturamento da Instituição HSCM. Com os dados obtidos pode-se analisar cada caso, separadamente, mediante grau de importância, cuidado e de valores.

\section{RESULTADOS E DISCUSSÕES}

Os dados a seguir chamaram a atenção quanto à desproporcionalidade das taxas de internações masculinas e femininas. Embora não tenha sido este objeto de estudo, porém, merece destaque e aponta para pesquisas futuras.

De acordo com a Tabela 1 e Gráfico 1, os resultados nos mostram a valorização que as pacientes do sexo feminino dão à saúde, bem como os cuidados que as mesmas dispensam consigo mesmas. Também evidenciam que os pacientes do sexo masculino ainda cultivam tabus sobre os cuidados com a saúde, bem como, pela busca de tratamento adequado e preciso quando necessário. 
Tabela 1 - Distribuição das internações quanto ao sexo

\begin{tabular}{|c|c|c|c|c|}
\hline Ano & Meses & Pacientes & Quantidade & Total \\
\hline \multirow{2}{*}{2007} & \multirow{2}{*}{ Janeiro a Fevereiro } & Feminino & 1109 & \multirow{2}{*}{1717} \\
\hline & & Masculino & 608 & \\
\hline Ano & Meses & Pacientes & Quantidade & Total \\
\hline \multirow{2}{*}{2008} & \multirow{2}{*}{ Janeiro a Fevereiro } & Feminino & 1157 & \multirow{2}{*}{1671} \\
\hline & & Masculino & 514 & \\
\hline Ano & Meses & Pacientes & Quantidade & Total \\
\hline \multirow{2}{*}{2009} & \multirow{2}{*}{ Janeiro a Fevereiro } & Feminino & 1199 & \multirow{2}{*}{1677} \\
\hline & & Masculino & 478 & \\
\hline Ano & Meses & Pacientes & Quantidade & Total \\
\hline \multirow{2}{*}{2010} & \multirow{2}{*}{ Janeiro a Agosto } & Feminino & 685 & \multirow{2}{*}{942} \\
\hline & & Masculino & 257 & \\
\hline \multicolumn{4}{|c|}{ Total Geral de Prontuários Médicos } & 6007 \\
\hline
\end{tabular}

Fonte: resultado da pesquisa

\section{Gráfico 1 - Caracterização gráfica dos prontuários avaliados quanto ao sexo dos pacientes internado no período}

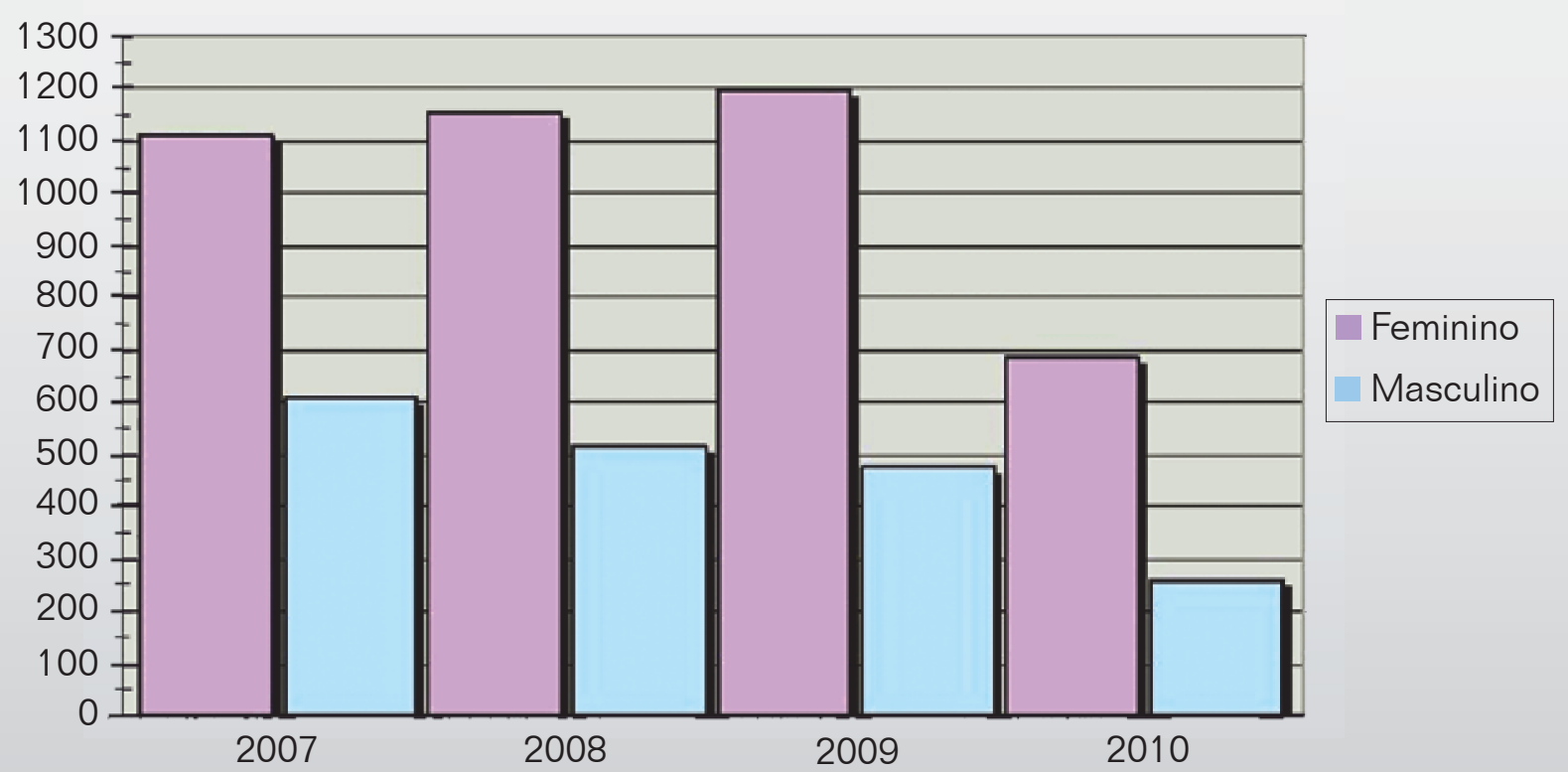

Fonte: Relatórios de auditoria Interna no HSCM.

\subsection{Avaliação de prontuários}

Durante todo o período de avaliação e verificação dos prontuários do HSCM, realizou-se uma detalhada e minuciosa consulta sobre o preenchimento dos mesmos, visando à constatação de quais seriam os prontuários devidamente completos e quais prontuários estavam com campos deficitários de preenchimentos.

De acrodo com o Gráfico 2, os prontuários incompletos, surpreendentemente, estavam em número superior aos prontuários completos. Isto posto, verifica-se a necessidade de conscientização e profissionalismo por parte dos profissionais da saúde que são responsáveis pelo setor de prontuários da instituição. 
Gráfico 2 - Avaliação dos prontuários quanto ao seu preenchimento:

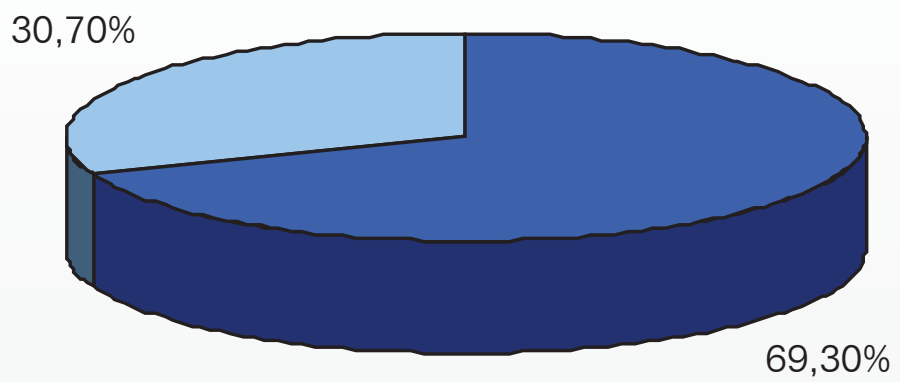

Prontuários Completos

Prontuários Incompletos

$69,30 \%$

Fonte: Relatórios de Auditoria Interna no HSCM

Tabela 2 - Caracterização dos problemas encontrados quanto ao preenchimento

\begin{tabular}{|c|c|c|}
\hline Inconformidades & Quantidade & Porcentagem Equivalente \\
\hline Checagem de Medicamentos & 913 & $49,51 \%$ \\
\hline Alta de Enfermagem & 236 & $12,79 \%$ \\
\hline Alta Médica & 128 & $6,94 \%$ \\
\hline Evolução Médica (Alta) & 103 & $5,60 \%$ \\
\hline Evolução de Enfermagem (Alta) & 122 & $6,61 \%$ \\
\hline Laudos de Radiografias & 96 & $5,21 \%$ \\
\hline Prescrição de exames realizados & 117 & $6,35 \%$ \\
\hline Coleta incompleta de dados dos pacientes & 129 & $6,99 \%$ \\
\hline Total & 1.844 & $100 \%$ \\
\hline
\end{tabular}

Gráfico 3 - Representação gráfica percentual das falhas encontradas no preenchimento dos prontuários

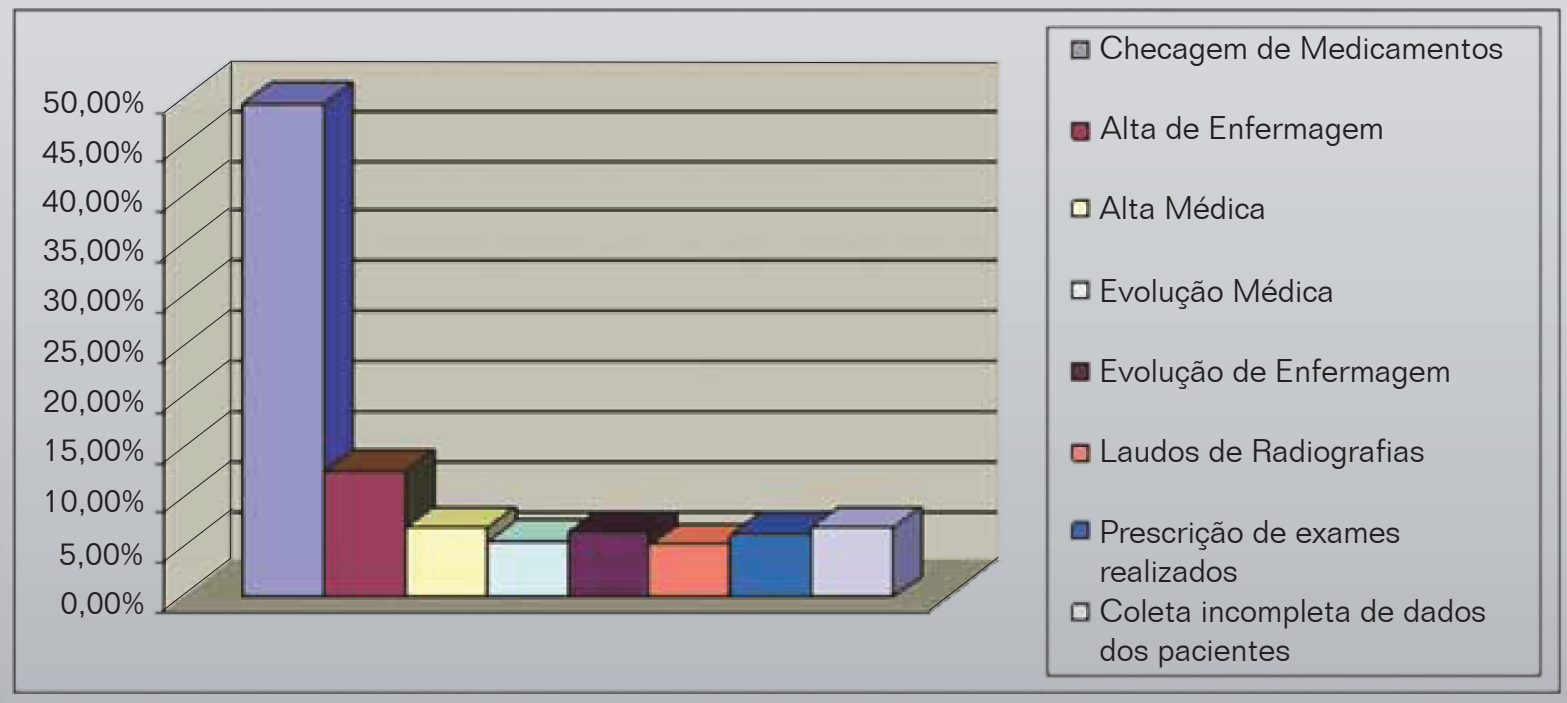

Fonte: Relatórios de auditoria interna no HSCM 


\title{
2.2 Checagem de medicamentos
}

O prontuário é de fundamental importância para a Auditoria nos Serviços de Saúde, bem como, para a gestão hospitalar baseada nas informações e checagem contidas nos mesmos. Sem ele, não é possível o hospital se adequar ao conceito de administração da saúde que diz:

\begin{abstract}
"Administração de saúde é planejar, organizar, dirigir, controlar, coordenar e avaliar os recursos e procedimentos pelos quais a demanda por cuidados médicos e de saúde, e as necessidades de um ambiente saudável são atendidos, mediante a provisão de serviços a clientes individuais." (AUSTIN 1974 apud MEZOMO, 2001 p.14)
\end{abstract}

Observou-se que a grande falta de checagem de medicamento ocasionou em perdas significativas, pois os medicamentos são dispensados pela farmácia. Porém quando os mesmos vão ser conferidos pelo responsável para a devida cobrança mediante a checagem, não conferem. Em pesquisa com alguns dos técnicos de enfermagem do setor foi verificado que o fato que mais persiste é a administração do medicamento no paciente em momento adverso à checagem no prontuário. Isso, na maioria das vezes, passa despercebido pelos técnicos, que trabalham em escala $12 / 36 \mathrm{hs}$, dificultando ainda mais a checagem posterior à administração do mesmo. Também relataram que conforme a gravidade do caso, a quantidade de paciente internado e o número de pessoas trabalhando, nem sempre acontece a checaem de medicamento no mesmo momentoEmbora o COREN e o CRM oriente para que não ocorra, há também os casos que os técnicos fazem contato telefônico com o médico responsável pelo paciente, caso aconteça alguma intercorrência, e o mesmo prescreve verbalmente algum medicamento sendo este administrado no mesmo instante, deixando a checagem para ser realizada no ato da prescrição deste pelo médico no prontuário, o que na maioria das vezes não ocorre. Há também o fato do esquecimento da checagem do prontuário por causa da sobrecargade trabalho e com muitos pacientes internados, paciente na urgência/ emergência. Assim, eles preocupam mais com o estado do paciente do que com o procedimento de checagem no prontuário.

Percebeu-se uma grande resistência por parte dos técnicos de enfermagem em seguir as orientações do enfermeiro e da administração, pois a maioria destes tem mais de 10 anos que trabalham na instituição e se acomodaram com a realização do trabalho. Tal fato afeta a segurança dos profissionais que ali trabalham perante os conselhos competentes de cada especialidade, bem como o faturamento da Instituição.

O aspecto humano do cuidado de enfermagem, com certeza, é um dos pontos mais difíceis de ser monitorado. Visar a rotina diária e complexa que envolve o ambiente do trabalho faz com que os técnicos em enfermagem tendam ,primeiramente, administrar a medicação para posteriormente checá-la como assim o deve ser feito. Porém o esquecimento e a sobrecarga de trabalho são grandes obstáculos a serem derrotados para o bom funcionamento do setor.

\subsection{Alta de enfermagem}

Em parte dos prontuários foi verificada a falta da evolução da alta do paciente por parte da equipe de enfermagem. A alta estava relatada pelo médico tanto na prescrição como na evolução. Porém faltava o relato da enfermagem informando em quais condições o paciente saiu de alta do hospital. Informaramme que este fato ocorre, na maioria das vezes em pacientes obstétricos, pois o obstetra passa e libera a alta para a mãe e o RN fica à espera do pediatra, que às vezes olha o paciente e orienta a liberá-lo após algumas horas, deixando para colocar a alta após a saída do mesmo do hospital, porém o paciente vai embora, o pediatra prescreve a alta,a enfermagem dispersa e não relata a saída do mesmo. Há também os casos em que o médico passa pela manhã, antes de ir para o plantão em outro município, avalia e prescreve para o dia, porém no final da tarde liga e pergunta sobre o estado em que o paciente se encontra. Tendo o mesmo evoluído com boa melhora, o profissional médico libera verbalmente o paciente deixando para prescrever a alta no momento em que retornar ao hospital, o que na maioria das vezes não é relatado pela enfermagem. Outro fato atípico é o acompanhamento do paciente em transferência, em que o médico acompanha o paciente e relata a transferência do mesmo ao retornar ao hospital, mas às vezes acontece de a equipe de enfermagem não verificar esta prescrição e deixar de evoluir esta alta por transferência.

Há também os casos em que o profissional médico coloca alta na prescrição e se esquece de relatar a mesma na evolução e vice-versa. 
Constata-se que:

A prescrição médica deverá gerar um amplo sistema de processamento, envolvendo setores amplamente diversificados do hospital: de inicio o serviço de enfermagem para conhecer e aplicar os recursos de toda natureza, prescritos pelo médico, e também o serviço de nutrição e dietética, para preparação de eventuais dietas especiais, bem como os setores complementares de diagnóstico e tratamento, para os quais o doente pode estar sendo encaminhado. (GONÇALVES, 1998, p.83)

A realidade aponta para a necessidade de compromisso por parte da equipe de enfermagem para com os prontuários dos pacientes, bem como a assistência profissional precisa, o carinho indispensável, a atenção, além da responsabilidade essencial e indiscutível, o que repercutirá em uma assistência com qualidade e eficiência.

\title{
2.4 Alta médica
}

Os casos em que não estava prescrita a alta médica foram em prontuários que o profissional médico relatou somente a alta na evolução, deixando de relatar a mesma na prescrição, ficando assim o prontuário incompleto, necessitando de atualização e complementação da prescrição médica. É necessário um segmento verídico do Organograma Horizontal, pois assim sendo, caso um profissional da enfermagem perceba que o colega, por qualquer motivo que seja, esqueceu-se de relatar a alta do paciente, pode o mesmo avisá-lo, para que assim a equipe não permaneça no erro.

\begin{abstract}
"Na integração da equipe são fundamentais a valorização e o respeito entre os profissionais, ocorrendo assim um reflexo positivo na relação entre os mesmos. Quando esta integração acontece, o cliente/paciente sente-se mais confiante, seguro e mais tranqüilo no que se refere a cuidados prestados por toda equipe, ocorrendo assim uma diminuição da ansiedade e proporcionando um ambiente hospitalar mais esperançoso." (GELAIN, 1963)
\end{abstract}

\subsection{Evolução médica e de enfermagem}

Certos prontuários encontravam-se com evoluções médicas incompletas, ou seja, o inverso do que ocorre quando o profissional médico não relata a alta médica na evolução do prontuário do paciente, onde a alta somente é relatada na prescrição, ficando a evolução incompleta. O profissional médico ao relatar tal procedimento, ou quaisquer outros vindouros da história clínica do paciente, é necessário estar prescrevendo e completando tanto a evolução quanto a prescrição médica para que o prontuário do paciente fique devidamente apresentável.

Houve também prontuários em que os técnicos de enfermagem se esqueceram de colocar informações importantes como a data na evolução, outros somente relataram dados vitais deixando de descrever a história clínica do paciente, ou seja, checagem incompleta. O que mais ocorreu foi à falta de informações importantes e necessárias para convalidar o diagnóstico com o procedimento solicitado, pois a evolução da enfermagem é de extrema importância para analisar se o diagnóstico informado pelo médico está em conformidade com o tratamento proposto, com os sintomas apresentados pelo paciente bem como a resposta do mesmo ao tratamento oferecido.

Anotações de Enfermagem - COREN - SP - Plenário 2008-2011 relata que:

"Os registros efetuados pela equipe de enfermagem (Enfermeiro, Técnico e Auxiliar de Enfermagem) têm finalidade essencial de fornecer informações sobre assistência prestada, assegurar comunicação entre os membros da equipe e saúde garantir continuidade das informações nas 24 horas, condição indispensável para compreensão do paciente de modo global. Os registros realizados no prontuário do paciente tornamse um documento legal de defesa dos profissionais, devendo, portanto, estar imbuídos de autenticidade e significado legal. Os mesmos refletem todo o empenho e força de trabalho da equipe de enfermagem, valorizando, assim, suas ações." 


\subsection{Prescrição de exames realizados / laudos de radiografias}

Prontuários continham exames laboratoriais, cujas datas estavam dentro do período de internação, porém os mesmos não estavam prescritos pelos médicos. Os técnicos de enfermagem alegaram que algumas vezes os exames são pedidos verbalmente, diretamente no laboratório onde os médicos conferem os resultados e se esquecem de prescrevê-los. Houve também raros prontuários em que aconteceu o contrário, o médico prescreveu os exames, o responsável pelo laboratório checou a prescrição, porém os resultados não se encontravam anexados ao prontuário. Foi relatado que este fato ocorre principalmente quando o paciente é transferido para outra unidade de saúde onde o médico entrega os exames ao paciente para que ele apresente estes como prova diagnóstica, ao dar entrada em outro município. Porém se esquecem de pedir uma cópia ao laboratório, deixando assim o prontuário incompleto.

Em alguns prontuários havia a prescrição do exame de $\mathrm{Rx}$, checado pelo técnico responsável pelo setor. Porém o laudo com a descrição do resultado do exame não se encontrava anexo ao prontuário. $\mathrm{O}$ correto é manter ou o laudo no prontuário ou o exame, como radiografias semdobras ou amassos, informando o resultado do exame realizado como prova diagnóstica. Devemos observar cuidadosamente os equívocos ocorridos até a presente data, para não mais repeti-los no desencadear de nosso trabalho, talvez um plano eficaz, um projeto certeiro ou quem sabe uma simples rotina, mas que proporcione um progresso significativo no segmento dos trabalhos e no pleno funcionamento do setor.

Para esses autores:

"Não se espera necessariamente que o futuro represente um progresso em relação ao passado, e tampouco acredita que seja extrapolável. Portanto, como primeira medida, é feita uma análise das perspectivas da empresa, identificando-se tendências, ameaças, oportunidades e descontinuidades singulares que possam alterar as tendências históricas". (ANSOFF e MCDONNELL, 1993, p. 38).

\subsection{Coleta incompleta de dados do pacientes}

A coleta incompleta de dados dos pacientes ocorreu, principalmente, ao paciente internado em horário noturno. Muitas vezes o paciente chega sem condições de relatar seus dados, não está portando seus documentos pessoais ou o acompanhante não sabe informar com precisão as informações necessárias. Também há casos em que é um urgência/emergência e, os familiares nervosos e preocupados pedem para passar os dados após o atendimento. Algumas vezes, a informação na é repassada na troca do plantão e o prontuário fica incompleto.

$\mathrm{Na}$ maioria das vezes o faturista liga para o paciente tentando completar a ficha, porém nem sempre consegue obter todas as informações necessárias para o devido preenchimento da ficha de admissão. Novamente verifica-se a importância do trabalho em equipe, do convívio harmonioso e da comunicação indispensável entre os setores.

\section{CONSIDERAÇÕES FINAIS}

Mediante pesquisa, avaliação e revisão nos prontuários médicos hospitalares, no período de 05/04/2010 a $31 / 08 / 2010$, constatou-se quão importante é o trabalho realizado sob a forma de uma equipe multiprofissional em um hospital, bem como o assessoramento e especialização dos profissionais da área de Auditoria em Sistemas de Saúde.

A referida análise mostrou que o tipo de organograma a ser seguido por uma Instituição hospitalar, no setor de enfermagem principalmente, deve ser o Organograma Horizontal onde os profissionais são dispostos um ao lado do outro de forma que, ao final do sistema forma-se um círculo, um elo, no qual fique evidenciado que se trata de uma "equipe" responsável pelo desenvolvimento o trabalho.

Os profissionais de enfermagem, administração e auditoria não tem como realizar seu trabalho de forma precisa, eficaz e eficiente, antes de aprender como ser inteiros consigo mesmos. A convivência tanto com o paciente quanto com os colegas de trabalho, nunca é neutra, pois, sempre carregamos conosco os preconceitos, valores, atitudes, enfim, nosso sistema de significados culturais. Por isso, é extremamente importante cuidar de quem cuida e acima de qualquer coisa valorizar e enfatizar o trabalho em equipe. 
Mas também podemos vivenciar que tanto a equipe de enfermagem como a equipe médica de um hospital são os grandes responsáveis pela melhoria das condições de vida e saúde de seus pacientes. E esta melhoria deve estar sempre ligada à qualidade do serviço prestado e a eficiência nas prescrições e relatos nos referidos prontuários.

A Auditoria remete à Instituição uma visão de auxílio, segurança e controle. Seja porque as relações humanas e profissionais interligam-se de tal forma que o monitoramento profissional e qualificado, como é o caso dos Auditores em Sistemas de Saúde, se tornam precisos, para uma administração eficaz do tratamento oferecido e proposto ao paciente, bem como uma segurança nos padrões de faturamento e gerenciamento da referida Instituição.

Tem se falado muito no tema Auditoria em Prontuários Médicos, pois esta se torna necessária diante das exigências do mercado hospitalar, bem como para que os convênios possam estar efetuando o pagamento sob o serviço faturado com tranqüilidade, mediante os padrões exigidos e também para que as políticas e regulações de saúde existentes sejam efetivas e eficazes.

Os prontuários, uma vez completos, verificados e auditados mediante conformidade entre procedimento solicitado, diagnóstico proposto e tratamento oferecido, são instrumentos necessários e indispensáveis para comprovação da história hospitalar e clínica de cada paciente, bem como pode servir como documento comprobatório de legalidade e defesa da equipe multiprofissional em caso de necessidades futuras.

Admitir um paciente exige atenção, eficácia, eficiência e precisão, pois as informações contidas na referida ficha hospitalar, nortearão todos os setores nos quais o paciente necessitará estar passando.

É uma realidade a prática do cuidado técnico do paciente, porém um Serviço de Auditoria em Sistemas de Saúde apenas instrui como esta deve ser devidamente seguida e relatada, conforme formação de cada profissional em particular.

Durante a hospitalização, a falta do relato sobre o estado de saúde dos pacientes nos devidos prontuários médicos e sobre os procedimentos aos quais estão sendo submetidos, somente revela que a equipe necessita estar mais envolvida tanto com a Instituição, quanto com o paciente. É através deste envolvimento que o tratamento se efetiva e o faturamento compensa a Instituição pelo serviço prestado e oferecido.

O cuidado de enfermagem é o ponto chave da hospitalização, uma vez que permite estabelecer intervenções terapêuticas centradas no paciente. Portanto devemos fazer desse ponto tão importante um aliado, capaz de assegurar todo e qualquer procedimento dentro da unidade hospitalar sem causar danos e perdas.

A enfermagem deve tomar consciência de que aprimorar suas técnicas e as suas relações interpessoais, bem como a sua dedicação para com o paciente e Instituição, contribuirá para amenizar questões administrativas significantes, visto que as próprias condições institucionais oferecidas são fonte geradora de alívio do sofrimento e da dor de pessoas que anseiam à saúde e o bem-estar, bem como fonte de trabalho e sustento de várias famílias.

Verifica-se a necessidade, diante de poucas ações e vastas teorias, da reflexão acerca da prática assistencial e aprimoramento de conhecimentos, pois estes são primordiais e necessários, para que uma Instituição sobreviva acerca de tantos contratempos, bem como as perdas já existente ocasionada por confronto de tabelas de valores, glosas desnecessárias e falta de incentivos voltados à promoção da saúde. Desenvolver o trabalho em equipe e reconhecer a humildade do semelhante mediante uma falha, erro ou esquecimento é dádiva de poucos, porém dever de muitos. Todo este trabalho enfatizou a importância do serviço de uma equipe em uma Unidade Hospitalar, porém reforçando sempre que o profissionalismo está acima de todo e qualquer ato. 


\section{REFERÊNCIAS}

CHIAVENATO, Idalberto. In: SILVA, Sandra Honorato da; ORTIZ, Diley Cardoso Franco; SHIMIZU, Helena Eri, TOTH, Márcia. Auditoria em enfermagem: implantação e desenvolvimento em Hospital Universitário da Universidade de São Paulo. Revista Escola de Enfermagem da USP. São Paulo, 24 (2), p. 199-209, ago. 1990.

KURCGANT, Paulina. Administração em enfermagem. São Paulo, EPU: 1991.

RIBEIRO, Circe de Melo. Auditoria de serviços de Enfermagem. Revista Brasileira de Enfermagem, Brasília, 25 (4), p. $91-103$, jul.-set. 1972.

BRASIL. Ministério do Planejamento, Orçamento e Gestão. Programa de Melhoria da Qualidade dos Serviços Prestados ao Cidadão: Instrumento de Auto-avaliação da Gestão Pública, Brasília, 2000, $157 \mathrm{p}$.

GONÇALVES, Ernesto Lima. Gestão hospitalar: Administrando o hospital moderno. São Paulo: Saraiva. 2006.

GONÇALVES, Ernesto Lima. Estrutura organizacional do hospital moderno. RAE - Revista de administração de empresas. São Paulo. V. 38 n.1 p. 80-90. 1998.

ANSOFF. H. Igor; McDONNELL. Edward J. Implantando a administração estratégica. Tradução de Antonio Zoratto Sanvicente, Guilherme Ary Plonky. 2. ed. São Paulo: Atlas, 1993.

MEZOMO, Joao Catarin. Gestão da qualidade na saúde: princípios básicos. Barueri: Manole, 2001.

DIAS. Claudia. Pesquisa qualitativa: características gerais e referências. 2000. Disponível em: < http://www.dfi.ccet.ufms.br/prrosa/metodologia/qualitativa.pdf > Acesso em: 18 out. 2010.

OLIVEIRA, Silvio Luiz de. Tratado de metodologia científica: projetos de pesquisas, TGI, TCC, monografias, dissertações e teses. Revisão Maria Aparecida Bessana. 2. ed. São Paulo: Pioneira, 2004.

GELAIN, I.J. A Humanização do Hospital. Ver. Paul. Hosp., São Paulo, v.16, n.1, p. 3-7, jan. 1963.

COREN - SP - Anotações de Enfermagem - Plenário 2008-2011. Disponível em: < www.sobecc.org.br/ programacao/coren/anotacoes_enfermagem.pdf > Acesso: 18 out. 2010. 